\title{
Evidence of a Sub-Saturn around EPIC 211945201
}

\author{
Abhijit Chakraborty $^{1}$ (1) Arpita Roy $^{2}$ (1) Rishikesh Sharma $^{1}$ (10, Suvrath Mahadevan ${ }^{3,4}$ (1), Priyanka Chaturvedi ${ }^{1}$, \\ Neelam J. S. S. V Prasad ${ }^{1}$, and B. G. Anandarao ${ }^{1}$ \\ ${ }^{1}$ Astronomy \& Astrophysics Division, Physical Research Laboratory, Ahmedabad 380009, India; abhijit@prl.res.in \\ ${ }^{2}$ Department of Astronomy, California Institute of Technology, Pasadena, CA 91125, USA \\ ${ }^{3}$ Department of Astronomy \& Astrophysics, The Pennsylvania State University, University Park, PA 16802, USA \\ ${ }^{4}$ Center for Exoplanets and Habitable Worlds, The Pennsylvania State University, University Park, PA 16802, USA \\ Received 2018 April 9; revised 2018 May 8; accepted 2018 May 9; published 2018 June 8
}

\begin{abstract}
We report here strong evidence for a sub-Saturn around EPIC 211945201 and confirm its planetary nature. EPIC 211945201b was found to be a planetary candidate from $K 2$ photometry in Campaigns 5 and 16 , transiting a bright star ( $V_{\text {mag }}=10.15$, G0 spectral type) in a 19.492 day orbit. However, the photometric data combined with false positive probability calculations using VESPA was not sufficient to confirm the planetary scenario. Here, we present high-resolution spectroscopic follow up of the target using the PARAS spectrograph (19 radial velocity observations) over a time baseline of 420 days. We conclusively rule out the possibility of an eclipsing binary system and confirm the $2 \sigma$ detection of a sub-Saturn planet. The confirmed planet has a radius of $6.12 \pm 0.1 R_{\oplus}$, and a mass of $27_{-12.6}^{+14} M_{\oplus}$. We also place an upper limit on the mass (within the $3 \sigma$ confidence interval) at $42 M_{\oplus}$ above the nominal value. This results in the Saturn-like density of $0.65_{-0.30}^{+0.34} \mathrm{~g} \mathrm{~cm}^{-3}$. Based on the mass and radius, we provide a preliminary model-dependent estimate that the heavy element content is $60 \%-70 \%$ of the total mass. This detection is important as it adds to a sparse catalog of confirmed exoplanets with masses between 10 and $70 M_{\oplus}$ and radii between 4 and $8 R_{\oplus}$, whose masses and radii are measured to a precision of $50 \%$ or better (only 23 including this work).
\end{abstract}

Key words: planetary systems - stars: individual (EPIC 211945201) - techniques: photometric - techniques: radial velocities

\section{Introduction}

A large number of transiting exoplanets have been discovered by dedicated space based photometric missions such as CoRoT (Baglin et al. 2006), Kepler (Borucki et al. 2010), and $K 2$ (Howell et al. 2014), and then followed up with ground-based spectroscopic resources for mass measurement using the radial velocity (RV) technique. However, the canon is limited in the number of exoplanets with radii between 2 and $8 R_{\oplus}$ that also have masses measured with a precision of $50 \%$ or better. Subsequently, this has limited our understanding of the composition, evolutionary history, and diversity of a population of exoplanets that have been variously defined as super-Neptunes $\left(10-40 M_{\oplus}\right.$ and 2-6 $R_{\oplus}$, Barragán et al. 2016) or sub-Saturns $\left(10-70 M_{\oplus}\right.$ and 4-8 $R_{\oplus}$, Petigura et al. 2017).

In the absence of precise mass measurements, or rather prior to the engagement of ground-based spectroscopic resources, there are still confidence levels that can be placed on the detection of an exoplanet candidate. Statistical validation tools such as BLENDER (Torres et al. 2011), PASTIS (Díaz et al. 2014), and VESPA (Morton 2012), can provide higher confidence in the planetary scenario than false positive alternatives by calculating the authenticity of the transit signal, in conjunction with ancillary information about the system, using a Bayesian approach. For example, recent work by Mayo et al. (2018) confirmed 149 exoplanets and identified 275 planetary candidates (PC) based on the false positive probabilities (FPP) of the transit signature. However, there remain sources whose transit signals are inadequate to confirm the nature of the system - these are essential candidates for follow up with high-precision RV spectrographs. Combining the photometry with RV data allows us to determine the mass and radius of the exoplanet and hence, its density. Theories of internal structure and planet formation mechanism are increasingly better constrained as we expand the number of exoplanets with precise measurements of mass and radius (Lopez \& Fortney 2014, and references therein). Hence, ground-based $\mathrm{RV}$ follow up for mass determination, although quite resourcelimited, remains of extreme importance for understanding exoplanet demographics.

In this paper, we report evidence for the sub-Saturn nature of EPIC 211945201b, a PC observed in K2 Campaigns 5 and 16. In Sections 2 and 3, we present the $K 2$ photometry and the analysis of archival Keck $K$-band imaging data. Section 4 elaborates on the statistical validation procedures of the PC using the VESPA framework. In Section 5, we describe our follow-up campaign with the PARAS spectrograph and the corresponding RV analysis. Section 6 reports our final host star properties, and Section 7 describes the simultaneous fitting of RV and photometric data. We discuss our results in Section 8 and conclude in Section 9.

\section{K2 Photometry}

The NASA $K 2$ mission (Howell et al. 2014) observed the source EPIC 211945201 from 2015 April 27 to 2015 July 10 (73 days) and 2017 December 07 to 2018 February 25 (81 days) as a part of Campaign 5 and Campaign 16, respectively. This target was identified as a transiting PC system by Pope et al. (2016), Barros et al. (2016), Petigura et al. (2018), and Mayo et al. (2018) using the light curve from Campaign 5, while Yu et al. (2018) recently used campaign 16 data to declare it a highquality PC.

There have been several pipelines developed to correct the systematics from the $K 2$ light curve. Examples include $\mathrm{k} 2 \mathrm{sff}$ 

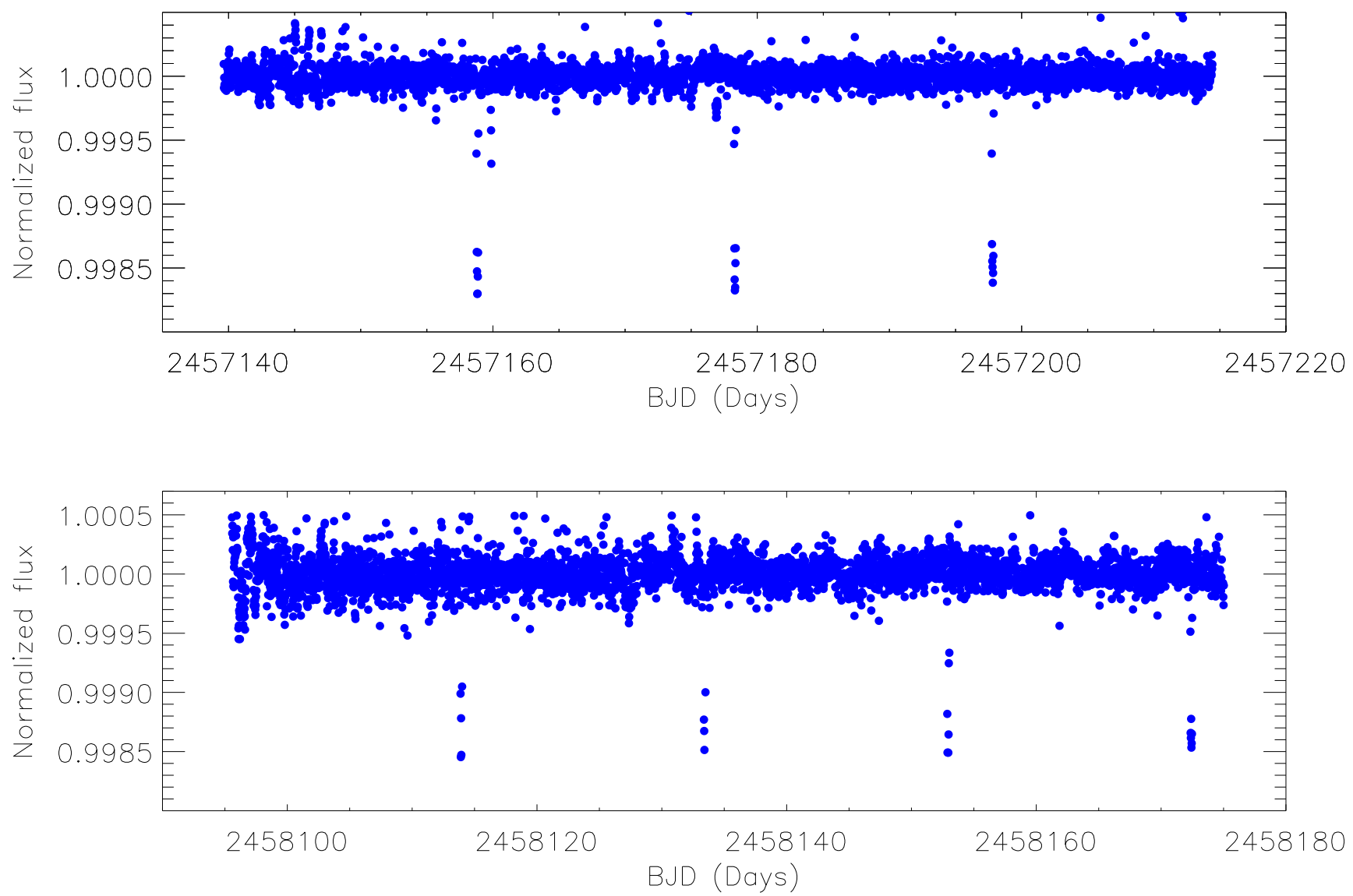

Figure 1. К2 РНО photometry data of EPIC 211945201. The upper panel shows the light curve from Campaign 5 ( $\approx 73$ days), while the lower panel light curve is from Campaign $16(\approx 81$ days). Three transits in Campaign 5 and four transits in Campaign 16 are clearly visible for this target.

(Vanderburg \& Johnson 2014), k2sc (Petigura et al. 2015; Aigrain et al. 2016), k2phot (Aigrain et al. 2016), and the everest package (Luger et al. 2016). All of the works identifying the possible planetary nature of EPIC 211945201b, referred to above, used one of above pipelines to extract and correct the light curve. In general, they first corrected the light curve and then searched for the significant transit signature using the BLS (Kovács et al. 2002) algorithm. In the BLS algorithm, the transit event is modeled as a box shaped modulation of the light curve. Following this approach, the occurrence of a single transit event was found at 19.49179 days (Mayo et al. 2018) from Campaign 5, and at 19.492036 days (Yu et al. 2018) from Campaign 16, which are both consistent within their error bars.

We retrieved the K2PHOT light curves (Petigura et al. 2015; Aigrain et al. 2016) for both campaigns through ExoFOP 5 these are shown in Figure 1. Seven transits, three in the upper panel and four in the lower panel, spaced every $\sim 19.49$ days, are clearly visible in the light curve of EPIC 211945201. In this work, we use the combined light curve of both Campaigns 5 and 16 and adopt a period of 19.49215 days from its analysis (see Section 7).

\section{High Angular Resolution Imaging}

High-resolution imaging is an immensely useful tool for constraining the probability of blended background objects. We made use of the archival high angular resolution AO-imaging

\footnotetext{
5 https://exofop.ipac.caltech.edu/k2/
}

data acquired using the Keck II/NIRC2 camera. This is also publicly available on ExoFOP (uploaded by user David Ciardi). The Keck AO data was obtained on 2016 January 21st (UT) with a $K$-band filter. NIRC2 has a pixel scale of 0 ".009942/ pixel. The observed image and $5 \sigma$ sensitivity curve are shown in Figure 2. The star appears single and has no close companions within the several arcseconds. At a separation of 0 . 5 , the estimated sensitivity to the companion is $\approx 8 \mathrm{mag}$. This effectively rules out the possibility of background sources within this separation contributing significant flux to the light curve. The estimated point-spread function (PSF) of the source is $0 . " 0526$.

\section{Statistical Validation}

We used the open source and publicly available $\mathrm{VESPA}^{6}$ package from Morton $(2012,2015)$ to determine the FPPs of the transit signature. The code uses transit parameters like shape, depth, and duration of each transit event, as well as independent observational constraints (like AO imaging for example) to validate the transit signal of a planet. VESPA uses TRILEGAL (TRIdimensional modeL of thE GALaxy) to simulate the population of each possible false positive scenario (such as background eclipsing binary (BEB), eclipsing binary (EB), hierarchical eclipsing binary (HEB) etc.) in a particular part of the sky using the coordinates of the source. It uses this population set combined with the observational constraints/ priors to calculate the prior for each scenario, and then the

\footnotetext{
6 https://github.com/timothydmorton/VESPA
} 


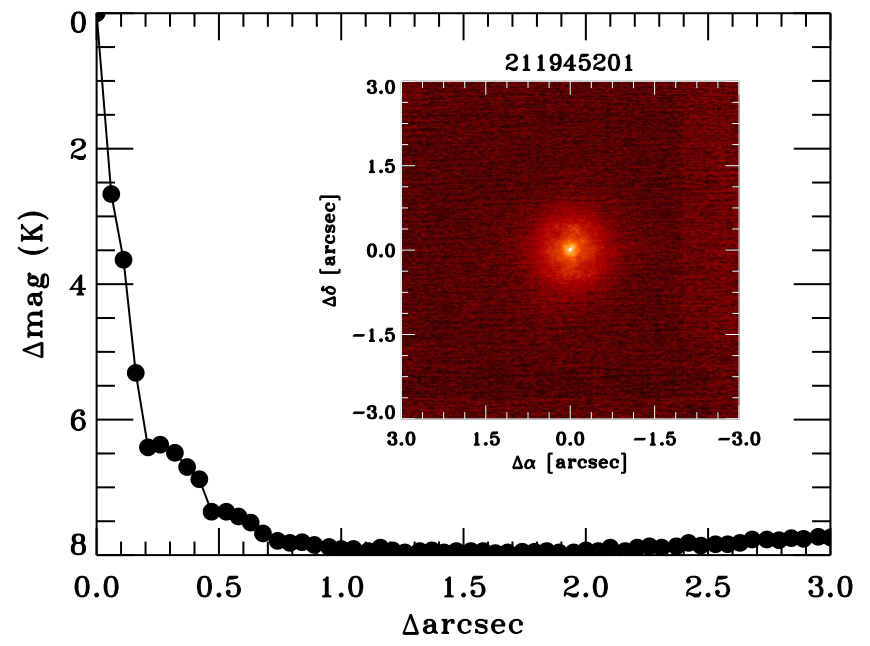

Figure 2. High angular resolution image of the source EPIC 211945201, and the $5 \sigma$ sensitivity/contrast curve in $K$-band observed with the NIRC2 instrument on the $10 \mathrm{~m}$ Keck II telescope (publicly available on ExoFOP, contributed by user David Ciardi).

likelihood of the each scenario. The resultant numbers are used to calculate a final probability for the validation of each contamination scenario.

VESPA requires certain inputs before calculating the FPP, such as aperture size used for the extraction of the light curve, secondary eclipse threshold, and photometric and spectroscopic parameters of the host star. Here, the default aperture size used for extraction of the K2 PHOT light curve (Petigura et al. 2015; Aigrain et al. 2016) is supplied to constrain the maximum allowed separation between the target and the source of the transit event. A search for the secondary eclipse was also undertaken using the method described in Dressing et al. (2017), between values of $0.3-0.8$ in orbital phase. It was found that the existing data could exclude events deeper than $5 \times 10^{-5}$ in units of the normalized flux. This value is then used by VESPA as a limit on the allowable secondary eclipse. High-resolution contrast curves can also be supplied to constrain the authenticity of transit signal. The Keck $K$-band contrast curve (Section 3) along with other publicly available contrast curves like Gemini $r$-band and Gemini $z$-band ${ }^{7}$ acquired using NIRI (Hodapp et al. 2003) are supplied as input. These contrast curves are especially useful for the validation process as they can preclude the presence of nearby or background stars above a certain brightness at a given distance on sky.

The properties of the host star from ExoFOP (e.g., coordinates, magnitudes in various bands, see Huber et al. 2016) and other parameters summarized in Table 1 were also supplied as input. The values of spectroscopic properties (mainly temperature, surface gravity, and metallicity) used for the FPP calculations were obtained through spectral analysis of data obtained with the PARAS spectrograph and are listed in Table 3 and further discussed in Section 6.

We performed the VESPA analysis separately for Campaign 5 and Campaign 16 data and found the probability for the planetary scenario to be $\sim 87 \%$ and $\sim 96 \%$, and the FPP to be $\sim 13 \%$ and $\sim 4 \%$, respectively. We note that much of this work was initially motivated by the individual results of Campaign 5, which were much less supportive of the planetary interpretation. Ultimately,

\footnotetext{
Also retrieved from ExoFOP, uploaded by David Ciardi.
}

Table 1

Stellar Parameters Supplied for FPP Calculations

\begin{tabular}{lcc}
\hline \hline Parameters & Value & Source \\
\hline Main Identifiers & & \\
$\alpha(J 2000)($ hh:mm:ss $)$ & $09: 06: 17.75$ & EPIC \\
$\delta(J 2000)$ (Degrees) & $19: 24: 08.11$ & EPIC \\
2MASS & J09061775+1924080 & EPIC \\
EPIC & 211945201 & EPIC \\
TYC & $1404-1186-1$ & EPIC \\
\hline Magnitudes & & \\
B & $10.937 \pm 0.080$ & EPIC \\
g & $10.479 \pm 0.030$ & EPIC \\
V & $10.154 \pm 0.056$ & EPIC \\
r & $10.038 \pm 0.040$ & EPIC \\
Kep & 10.115 & EPIC \\
i & $9.959 \pm 0.080$ & EPIC \\
J & $9.144 \pm 0.023$ & 2MASS \\
H & $8.908 \pm 0.028$ & $2 M A S S$ \\
K & $8.837 \pm 0.020$ & 2MASS \\
W1 & $8.822 \pm 0.023$ & WISE \\
W2 & $8.844 \pm 0.020$ & WISE \\
W3 & $8.810 \pm 0.028$ & WISE \\
W4 & $8.623 \pm 0.398$ & WISE \\
\hline
\end{tabular}

Note. Parameters with source flagged as EPIC are taken from the Ecliptic Plane Input Catalog available at https://archive.stsci.edu/k2/epic/search.php. Other parameters whose sources are flagged as 2MASS and WISE are taken from (Cutri et al. 2003) and (Cutri et al. 2013), respectively.

we used the combined light curve from both the campaigns to calculate a probability of $\sim 98 \%$ (Figure 3 ) for the planetary scenario, with the remaining $\sim 2 \%$ probability attributed to an EB scenario. The threshold for planetary validation is a stringent $<1 \%$ FPP (Crossfield et al. 2016), which relegated EPIC 211945201 to the status of PC, despite the coverage of multiple transit events. Because the results of the FPP calculation from VESPA could not single-handedly rule out the possibility of an EB, follow up with high-precision Doppler spectroscopy was necessary to establish the planetary nature of the candidate.

\section{Spectroscopic Follow-up Observations}

In order to confirm the planetary nature of the candidate, highresolution $(R \sim 67,000)$ spectroscopic follow-up observations were undertaken with the PARAS spectrograph (Chakraborty et al. 2014) mounted on a 1.2 meter telescope at Gurushikhar Observatory, Mount Abu, India. PARAS is a fiber-fed, temperature and pressure stabilized, white pupil echelle spectrometer that has earlier been shown to achieve $\sim 1 \mathrm{~m} \mathrm{~s}^{-1} \mathrm{RV}$ precision on timescales of a month (Roy et al. 2016). A total of 19 spectra were acquired between 2016 November 25th and 2018 January 18th using the simultaneous wavelength calibration mode (using a ThAr hollow cathode lamp) as explained in Chakraborty et al. (2014). Besides science exposures, five bias frames and three flat frames were also acquired on each night in order to correct the bias and verify the cross-dispersed order locations on the stabilized instrument. After each science exposure, ThAr-ThAr exposures were also acquired, illuminating both the science and calibration fiber with the ThAr lamp, to correct the instrumental as well as inter-fiber drift. Additional details of the spectrograph, observational procedure, and data analysis techniques are described in Chakraborty et al. (2014). 


\section{epic211945201}

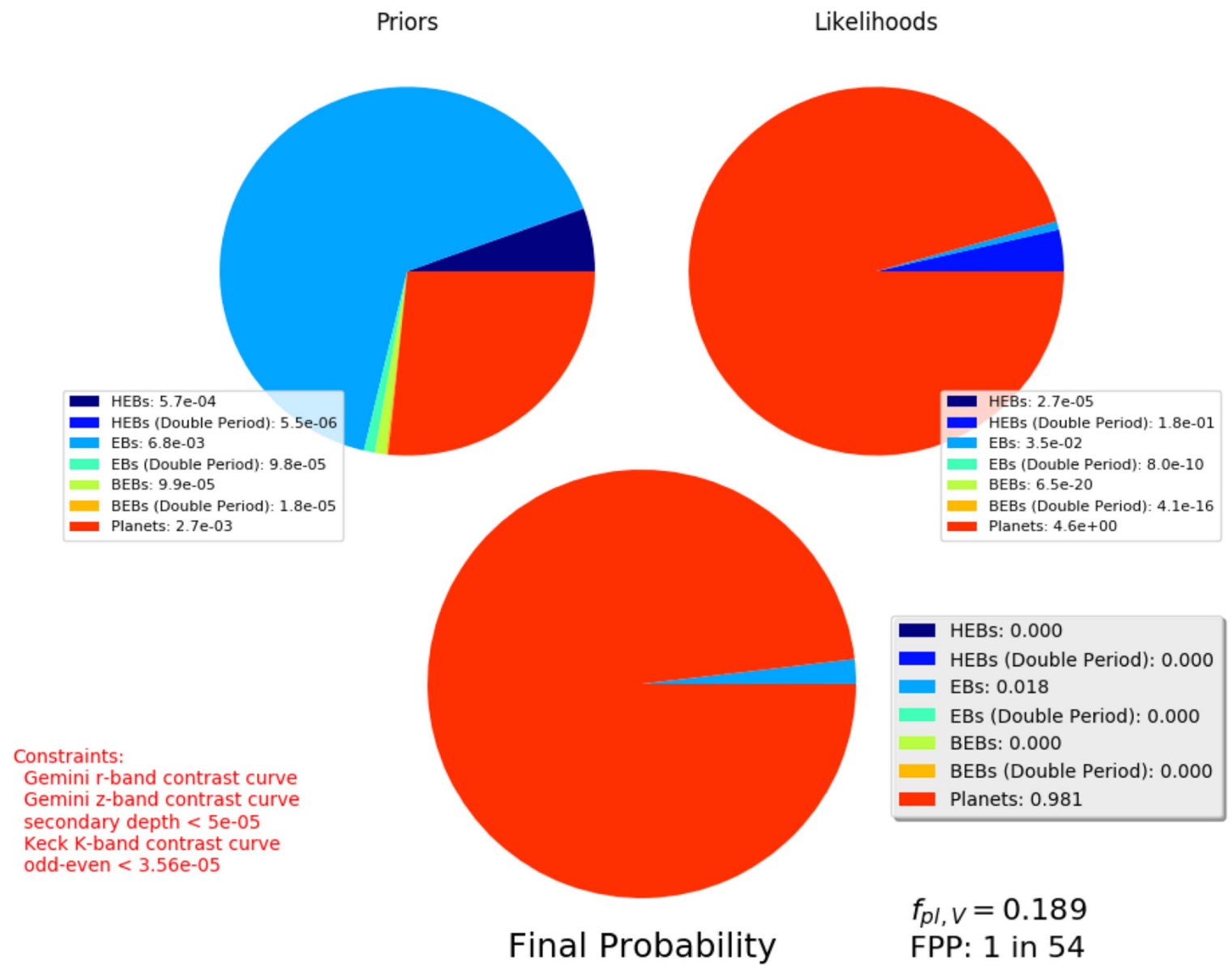

Figure 3. False Positive Probability (FPP) analysis using the VESPA package from Morton (2012, 2015). The incorporates the prior likelihood of a false positive scenario (given sky position, AO-imaging contrast curve data, and wavelength-dependent magnitudes), with the likelihood of the transit photometry under various scenarios. The results shown here are obtained by using the combined light curve of EPIC 211945201 from Campaigns 5 and 16.

The source was observed only in dark (moon-less) and photometric sky conditions. The airmass for all the epochs were $<1.5$ and seeing was better than 1 ." 5 . The exposure time for each observation was between 1800 and 3000s as the source is toward the fainter limit of PARAS (see Table 2), which resulted in signal-to-noise ratios $(\mathrm{S} / \mathrm{N})$ between 13 and 20 per pixel at the blaze peak wavelength of $5500 \AA$. A list of epochs and observational details is shown in Table 2. The first column in the table represents observation time stamp in terms of BJDTDB. The observed RV values are listed in the next column followed by the RV errors, which are based on both photon noise and the fitting errors of the cross-correlation function (CCF; for CCF error estimations, see Chaturvedi et al. 2016a). The RV data spans more than a year-nearly 420 days.

\subsection{RV Analysis}

The entire data reduction and RV analysis for PARAS was carried out by the automated pipeline in IDL based on the REDUCE optimal extraction routines of Piskunov \& Valenti (2002) and explained in Chakraborty et al. (2014). In order to reduce the data, the pipeline performs routine tasks like bias subtraction, order trace verification, cosmic ray correction
Table 2

Radial Velocities of EPIC 211945201 in Chronological Order

\begin{tabular}{lccrcc}
\hline \hline BJD $_{\text {TDB }}$ & $\begin{array}{c}\mathrm{RV} \\
\left(\mathrm{km} \mathrm{s}^{-1}\right)\end{array}$ & $\begin{array}{c}\sigma-\mathrm{RV} \\
\left(\mathrm{km} \mathrm{s}^{-1}\right)\end{array}$ & $\begin{array}{c}\mathrm{BIS} \\
\left(\mathrm{km} \mathrm{s}^{-1}\right)\end{array}$ & $\begin{array}{c}\sigma-\mathrm{BIS} \\
\left(\mathrm{km} \mathrm{s}^{-1}\right)\end{array}$ & $\begin{array}{c}\text { Exp. } \\
(\mathrm{s})\end{array}$ \\
\hline 2457717.469599 & 1.2657 & 0.0087 & 0.1763 & 0.0105 & 3000 \\
2457755.352017 & 1.2698 & 0.0088 & -0.0437 & 0.0155 & 3000 \\
2457756.343921 & 1.2613 & 0.0089 & -0.0066 & 0.0110 & 3000 \\
2457757.300892 & 1.2607 & 0.0098 & 0.3477 & 0.0102 & 3000 \\
2457757.344621 & 1.2678 & 0.0088 & 0.3419 & 0.0132 & 3000 \\
2457758.348027 & 1.2567 & 0.0097 & 0.1885 & 0.0099 & 3000 \\
2457761.483625 & 1.2594 & 0.0098 & -0.0853 & 0.0112 & 3000 \\
2457761.522539 & 1.2597 & 0.0086 & -0.1513 & 0.0107 & 3000 \\
2457786.395919 & 1.2501 & 0.0086 & 0.1565 & 0.0080 & 2400 \\
2457787.435365 & 1.2610 & 0.0098 & -0.1214 & 0.0086 & 2400 \\
2457790.417556 & 1.2487 & 0.0098 & 0.0058 & 0.0099 & 2400 \\
2457815.206300 & 1.2687 & 0.0123 & 0.3546 & 0.0244 & 1800 \\
2457816.230661 & 1.2671 & 0.0124 & 0.1758 & 0.0239 & 1800 \\
2457818.178771 & 1.2704 & 0.0121 & 0.1407 & 0.0127 & 1800 \\
2457843.222090 & 1.2525 & 0.0150 & 0.0891 & 0.0148 & 2400 \\
2458080.433400 & 1.2528 & 0.0151 & 0.1871 & 0.1255 & 2400 \\
2458083.416314 & 1.2720 & 0.0129 & 0.0690 & 0.1746 & 2400 \\
2458111.447097 & 1.2606 & 0.0102 & 0.1629 & 0.0422 & 2400 \\
2458137.379171 & 1.2628 & 0.0136 & 0.7929 & 0.1392 & 2400 \\
\hline
\end{tabular}


Table 3

Stellar Parameters

\begin{tabular}{lccc}
\hline \hline Parameters & (Petigura et al. 2018) & (Mayo et al. 2018) & This Work \\
\hline$T_{\text {eff }}[\mathrm{K}]$ & $6018 \pm 60$ & $6046 \pm 50$ & $6025 \pm 100$ \\
$\log (g)[\mathrm{dex}]$ & $4.13 \pm 0.1$ & $4.14 \pm 0.1$ & $4.25 \pm 0.1$ \\
{$[\mathrm{Fe} / \mathrm{H}][\mathrm{dex}]$} & $0.12 \pm 0.04$ & $0.05 \pm 0.08$ & $0.1 \pm 0.1$ \\
$v_{\text {micro }}\left[\mathrm{Km} \mathrm{s}^{-1}\right]$ & $\ldots$ & $\ldots$ & $0.4 \pm 0.1$ \\
$v_{\text {rot }}\left[\mathrm{Km} \mathrm{s}^{-1}\right]$ & $3.3 \pm 1.0$ & $\ldots$ & $4.0 \pm 1.0$ \\
\hline
\end{tabular}

(especially important due to the deep depletion CCD in PARAS), and optimal extraction of both target and calibration spectra. Instrumental drifts are tracked and corrected by crosscorrelation of the simultaneous calibration spectrum with a custom ThAr mask made for the PARAS lamp. RVs are derived by cross-correlating the target spectra with a suitable numerical stellar template mask. The stellar mask is created from a synthetic spectrum of the star, containing the majority of deep photospheric absorption lines. See Baranne et al. (1996), Pepe et al. (2002) and references therein for a more detailed description of the mask cross-correlation method. Based on the temperature given in Table 3, which is determined from spectral analysis (see Section 6), our source is found to be a F9/G0 spectral type star. Thus, we use a G2-type stellar mask for cross-correlation of the spectra. RV measurement errors, which are given in Table 2, are based on photon noise errors (Bouchy et al. 2001) and the errors associated with the CCF fitting function. These errors range from 9 to $16 \mathrm{~m} \mathrm{~s}^{-1}$; the method for their computation is described in Chaturvedi et al. (2016a).

The radial velocities from PARAS are plotted in Figure 6, along with the best-fit model (determined by the joint fit with the photometric data) described in Section 7. In order to check $\mathrm{RV}$ variation induced by a blended spectrum, we computed the bisector inverse slope (BIS) of the cross-correlation function for each observation in the manner of Queloz et al. (2001). The value of the BIS for each $\mathrm{CCF}$, and its respective errors, are listed in Table 2 and plotted in Figure 7. We find no correlation between the BIS and the measured RV. If the signal detected was due to a blended spectrum, then we would expect to see a strong correlation between the bisector slopes and RV measurements (e.g., Wright et al. 2013).

\subsection{Discarding an EB Scenario}

The radial velocities observed with PARAS are listed in Table 2 and plotted in Figure 4. The change in the RV values throughout our observational span is small-within $\pm 20 \mathrm{~m} \mathrm{~s}^{-1}$ - which discards the possibility that this is an EB system. The RV data set combined with FPP results (Section 4) shows very strong evidence that the body revolving around EPIC 211945201 in a 19.491 day orbit is indeed a planet.

To verify the instrument stability of the PARAS spectrograph over the same period of time, the RV standard star HD55575 (Bouchy et al. 2013) was monitored closely alongside EPIC 211945201. This star was observed by SOPHIE (Perruchot et al. 2008; Bouchy et al. 2013) in HR+ mode for a span of 118 days. The RV dispersion $\left(\sigma_{\mathrm{RV}}\right)$ of this target was reported to be $3.4 \mathrm{~m} \mathrm{~s}^{-1}$ in Bouchy et al. (2013). We acquired 58 spectra of HD55575 over a span of 391 days. The $\mathrm{RV}$ s were then calculated identical to the manner described in Section 5.1 for EPIC 211945201. The $\sigma_{\mathrm{RV}}$ with PARAS was found to be $3.5 \mathrm{~m} \mathrm{~s}^{-1}$. Nightly binning of the data points reduced this dispersion further to $3.1 \mathrm{~m} \mathrm{~s}^{-1}$. This demonstrates that our RV dispersion is consistent with that achieved by SOPHIE for HD55575, and quantifies the upper limit of the spectrograph's stability during the entire long span of observations of EPIC 211945201. With strong evidence that our source hosts an exoplanet, we proceed to estimating the mass of the transiting body by simultaneous modeling of RV and photometry data in the Section 7.

\section{Physical Parameters of the Star}

The spectral parameters of the star were first estimated using the PARAS SPEC package (Chaturvedi et al. 2016a). PARAS $\mathrm{SPEC}$ is a stellar synthesis pipeline that estimates $T_{\text {eff }}, \log (g)$, $[\mathrm{Fe} / \mathrm{H}], v \sin i$ and $v_{\text {micro }}$ by implementing both synthetic spectral fitting and equivalent width measurements. The details of the package can be found in Chaturvedi et al. (2016a) and Chaturvedi et al. (2016b). For EPIC 211945201, the coadded high $\mathrm{S} / \mathrm{N}$ spectra for all of the epochs listed in Table 2 were used for this analysis. The adopted values for stellar parameters are the weighted average of the results obtained from both the methods of PARAS SPEC. Stellar parameters present in the literature, along with our new estimates, are listed in Table 3. It can be seen that our estimated parameters are within the $2 \sigma$ confidence interval of the parameters available in the literature. These estimated stellar parameters, along with the photometric magnitudes given in Table 1 of the star in different bands, are used to derive the physical parameters of the star. The new precision astrometry from the GAIA DR2 data release (Gaia Collaboration et al. 2018) is also used in this derivation of stellar parameters. The parallax for the star measured by GAIA is $5.475 \pm 0.039 .^{8}$ The Dartmouth Stellar evolution database (Dotter et al. 2008) is utilized to obtain the radius, mass, age and distance to the host star using the ISOCHRONE package (Morton 2015). The uncertainties associated with photometric and spectroscopic observables were also taken into account while estimating the properties of the star. Finally, a radius of $R_{*}=1.38_{-0.018}^{+0.017} R_{\odot}$, mass of $M_{*}=1.18_{-0.04}^{+0.03} M_{\odot}$, age of $3.99_{-0.7}^{+0.85} \mathrm{Gyr}$, and a distance of $182.6 \pm 1.3 \mathrm{pc}$ are determined for the star. The very precise parallax measurements from GAIA lead to an extremely precise determination of stellar parameters, shrinking the error bars by an order of magnitude, and exhibiting the tremendous value of this mission.

\section{Simultaneous Fitting and Modeling of RV and Photometry Data}

The K2 photometry data and PARAS RV data are simultaneously fitted using the PYANETI routine of (Barragán et al. 2016, 2017). This PYTHON code uses Markov chain Monte Carlo (MCMC) methods with a Bayesian approach and a parallelized ensemble sampler algorithm in Fortran. The photometric data set included in the joint analysis was subset of the whole $\mathrm{k} 2 \mathrm{sff}$ light curve. About $13 \mathrm{hr}$ of data centered on each of the seven transits observed by $K 2$ is selected. The final data set contains 194 photometric data points and $19 \mathrm{RV}$ data points as listed in Table 2.

The data sets were fitted assuming a circular Keplerian orbit, i.e., we fixed eccentricity $e=0$ and longitude of periastron $\omega=90^{\circ}$. Other orbital parameters were allowed to float, including the systemic velocity $\gamma$ for the PARAS instrument,

\footnotetext{
https://gea.esac.esa.int/archive/
} 


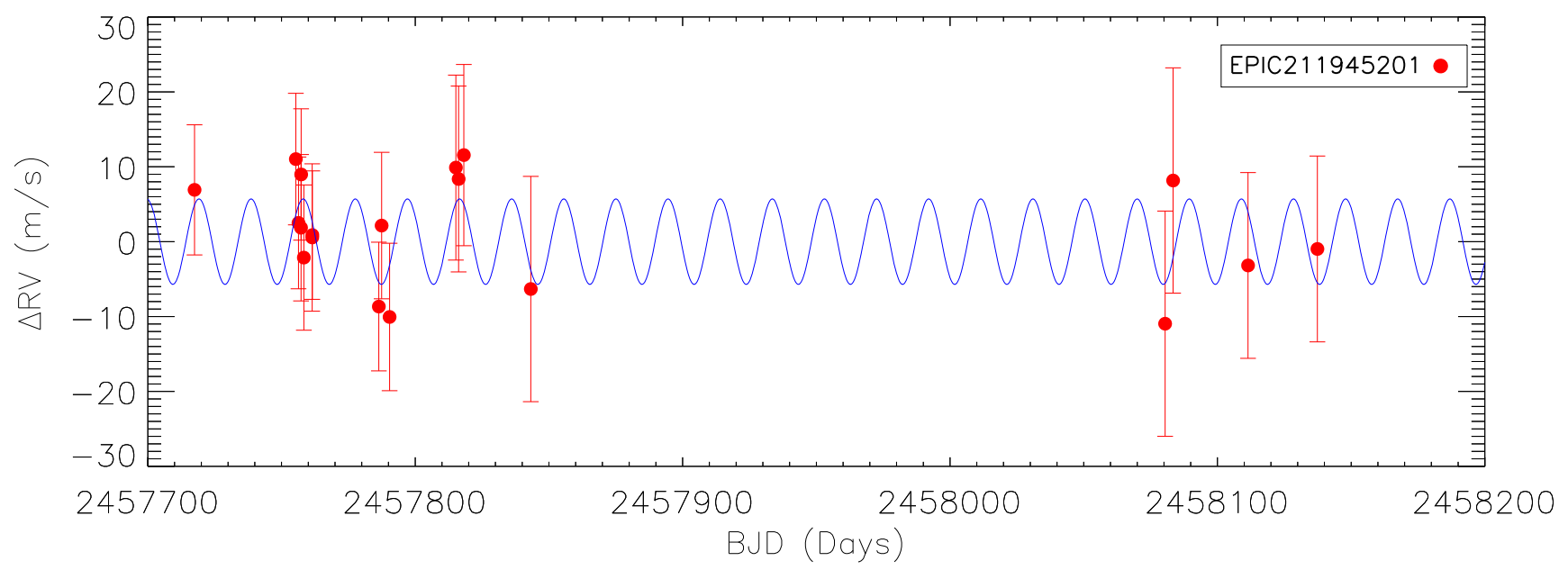

Figure 4. Radial velocities for EPIC 211945201 over a span of 420 days, observed with the PARAS spectrograph. The absence of large RV dispersion is clearly seen, which discards an EB scenario(see Section 5.2). The overlaid blue curve is the best-fit model to our data set (from PYANETI).

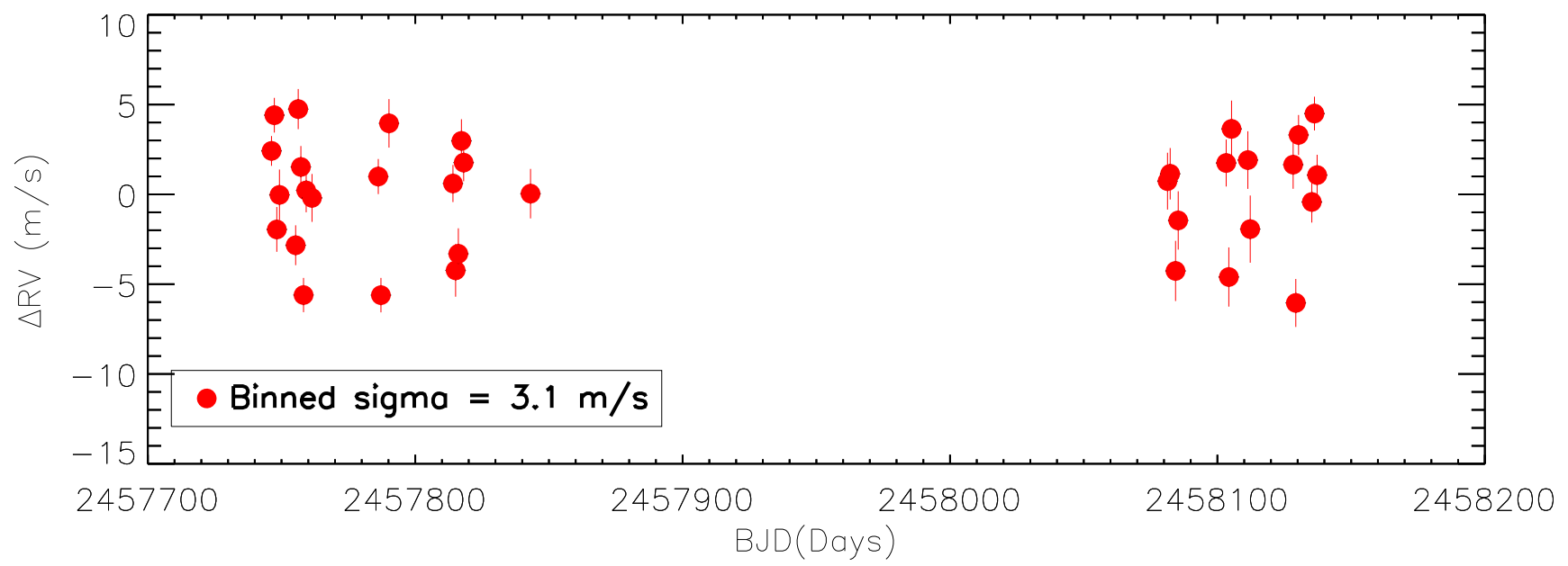

Figure 5. Radial velocities for standard star HD55575. The RV points in red show nightly binned averages. The data spans over 391 days and demonstrates instrument stability and performance during the acquisition of the EPIC 211945201 data.
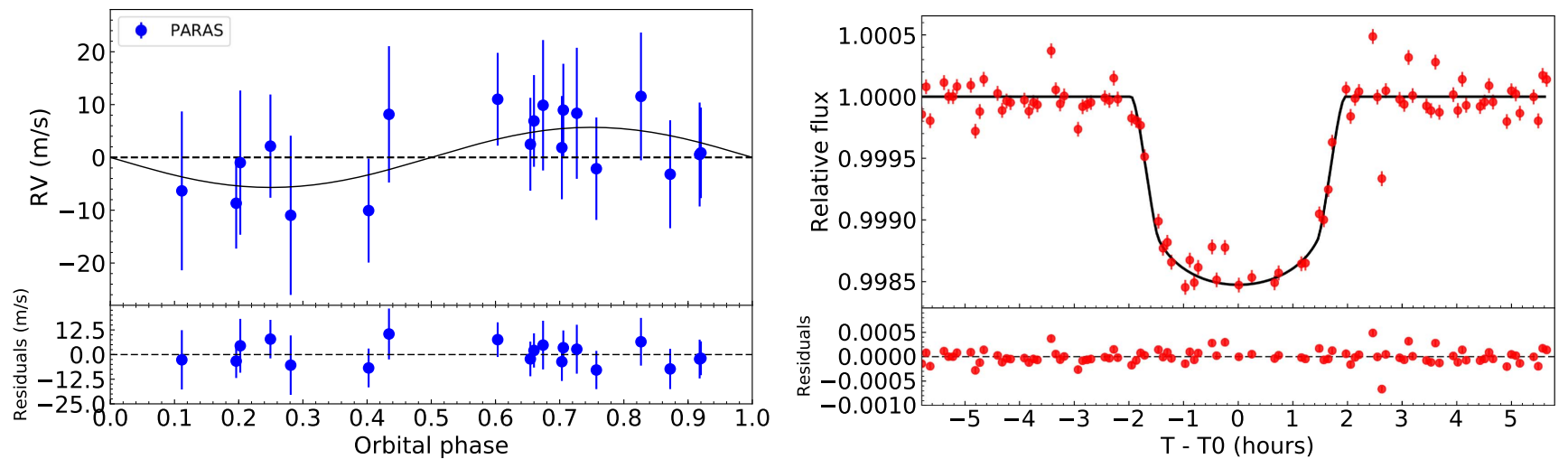

Figure 6. Left panel: RVs taken by PARAS, phased to the 19.491 days period as determined from the simultaneous fitting of the photometric and RV data using PYANETI. The best-fit model is also displayed together with the residuals. Right panel: phase folded K2PHOT light curve centered on the $T_{0}$ with the best-fit model in black overlaid.

the RV semi-amplitude $K$, mid-transit time $T_{0}$, orbital period $P_{\text {orb}}$, impact parameter $b$, semimajor axis in terms of stellar radius $a / R_{*}, q_{1}, q_{2}$, and the planet to star radius ratio $R_{p} / R_{*}$. Here, $q_{1}$ and $q_{2}$ are the parameterization of $u_{1}$ and $u_{2}$ as described in Kipping (2013). Also, in order to fit the photometry data, the quadratic limb-darkening law of Mandel \& Agol (2002) was followed.

We generated 250,000 independent points for each free parameter by exploration of parameter space using 500 Markov chains. In order to find the global solution for the data set, a 


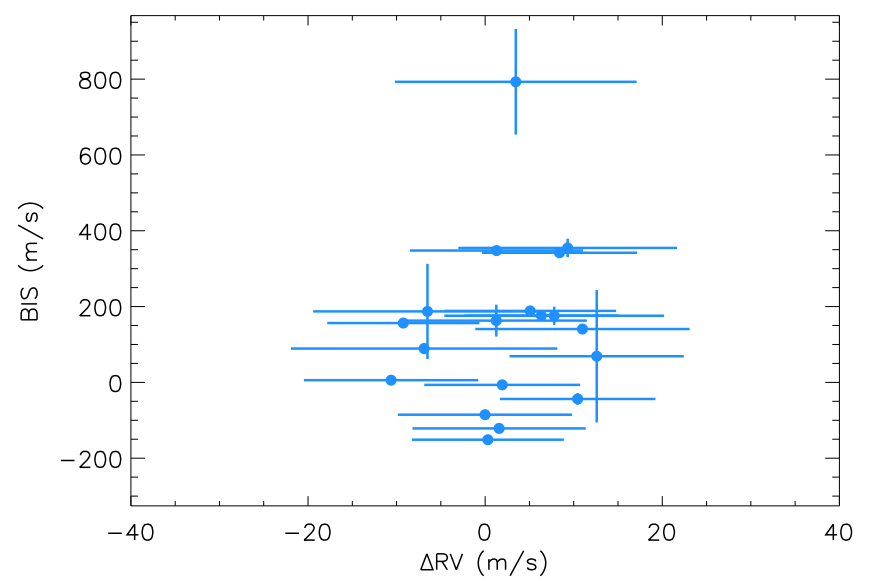

Figure 7. Distribution of measured radial velocities from PARAS, and associated values of the bisector inverse slope from the CCF. There is no significant correlation between the two, ruling out the possibility of blended spectra.

wide range of uniform priors $P_{\text {orb }}=[19.40,19.60]$ days, $T_{0}=[2458112.5403,2458114.6532], b=[0,1], a / R_{*}=$ $[5,100], R_{p} / R_{*}=[0.005,0.1], K=[0.001,1.0] \mathrm{km} \mathrm{s}^{-1}$, $\gamma_{j}=[1,100] \mathrm{km} \mathrm{s}^{-1}$ and $q_{1}, q_{2}=[0,1]$ were chosen. The final derived parameter values and their associated uncertainties are given by the median and $68.3 \%$ confidence interval of the posterior probability distribution; these are listed in Table 4. The global fitting of the limb-darkening coefficients gives $q_{1}=0.149_{-0.075}^{+0.077}$ and $q_{2}=0.457_{-0.308}^{+0.384}$, which results in $u_{1}=0.327_{-0.231}^{+0.345}$ and $u_{2}=0.028_{-0.289}^{+0.220}$, respectively. As the uncertainties associated with these are very high, we instead interpolate the limb-darkening coefficients given in Claret \& Bloemen (2011) for the Kepler-band and also list these values in Table 4.

\section{Discussion}

The simultaneous fitting of RV and photometric data gives a RV semi-amplitude of $K=5.7_{-2.7}^{+3.0} \mathrm{~m} \mathrm{~s}^{-1}$, and mass of $M_{p}=27_{-12.6}^{+14} M_{\oplus}$ for EPIC $211945201 \mathrm{~b}$. This gives a $3 \sigma$ upper limit on the mass of $42 M_{\oplus}$ above the nominal value. Leveraging the precision of the newly released GAIA DR2 data, the radius of the planet is derived to be $6.12 \pm 0.10 R_{\oplus}$. This radius and mass correspond to a density of $0.65_{-0.30}^{+0.34} \mathrm{~g} \mathrm{~cm}^{-3}$. It is to be noted here that earlier radius estimates of $6.0_{-0.8}^{+0.9} R_{\oplus}$ by Petigura et al. (2018) and $5.85_{-0.78}^{+0.95} R_{\oplus}$ by Mayo et al. (2018) from Campaign 5 transit data alone; and $5.3 R_{\oplus}$ by Yu et al. (2018) from Campaign 16 data, are in agreement with our simultaneous fitting results. The planet thus has a Saturn-like density while its mass and radius place it in the super-Neptune or sub-Saturn classification of exoplanets. This class of planets is currently very underpopulated (Bonomo et al. 2014).

EPIC 211945201b lies in the overlapping region between super-Neptunes and sub-Saturns. There are only 22 sub-Saturns in the range of $4-8 R_{\oplus}$ whose mass and radius are precisely known, as discussed and listed in Table 7 of Petigura et al. (2017). In spite of the small range in radii, these planets are found to have a large mass range, and thus densities that vary between 2.40 and $0.26 \mathrm{~g} \mathrm{~cm}^{-3}$, indicating diverse formation mechanisms (Bayliss et al. 2015). There is no obvious correlation between the radius and mass of these planets,
Table 4

Median Values and 68\% Confidence Intervals for the Light-curve and Radial Velocity Parameters of the Epic 211945201 System

\begin{tabular}{|c|c|c|}
\hline Parameter & Units & Value \\
\hline \multicolumn{3}{|c|}{ Stellar Parameters: } \\
\hline$M_{*}$ & Mass $\left(M_{\odot}\right)$ & $1.18_{-0.04}^{+0.03}$ \\
\hline$R_{*}$ & Radius $\left(R_{\odot}\right)$ & $1.38_{-0.018}^{+0.017}$ \\
\hline$\rho_{*}$ & Density (cgs) & $0.63_{-0.03}^{+0.04}$ \\
\hline $\log \left(g_{*}\right)$ & Surface gravity (cgs) & $4.25 \pm 0.1$ \\
\hline$T_{\text {eff }}$ & Effective temperature $(\mathrm{K})$ & $6025 \pm 100$ \\
\hline$[\mathrm{Fe} / \mathrm{H}]$ & Metallicity & $0.1 \pm 0.1$ \\
\hline Age & Gyr & $3.99_{-0.7}^{+0.85}$ \\
\hline Distance & $\mathrm{pc}$ & $182.6 \pm 1.3$ \\
\hline$v_{\text {micro }}$ & Microturbulence $\left(\mathrm{Km} \mathrm{s}^{-1}\right)$ & $0.4 \pm 0.1$ \\
\hline$v_{\text {rot }}$ & Rotational Velocity $\left(\mathrm{Km} \mathrm{s}^{-1}\right)$ & $4.0 \pm 1.0$ \\
\hline \multicolumn{3}{|c|}{ Planetary Parameters: Fitted } \\
\hline$T_{0}$ & Time of transit $\left(\mathrm{BJD}_{\mathrm{TDB}}\right)$ & $2458113.93994_{-0.00039}^{+0.00039}$ \\
\hline$e$ & Eccentricity & 0.0 (fixed) \\
\hline$\omega_{*}$ & Argument of periastron (degrees) & 90.0 (fixed) \\
\hline$P$ & Period (days) & $19.49213_{-0.00001}^{+0.00001}$ \\
\hline$b$ & Impact Parameter & $0.85_{-0.008}^{+0.007}$ \\
\hline$a / R_{*}$ & Semimajor axis in stellar radii & $23.10_{-0.47}^{+0.47}$ \\
\hline$R_{P} / R_{*}$ & Radius of planet in stellar radii & $0.0407_{-0.0003}^{+0.0003}$ \\
\hline$K$ & RV semi-amplitude $\left(\mathrm{m} \mathrm{s}^{-1}\right)$ & $5.7_{-2.7}^{+3.0}$ \\
\hline \multicolumn{3}{|c|}{ Planetary Parameters: Derived } \\
\hline$i$ & Inclination (degrees) & $87.90 \pm 0.06$ \\
\hline$a$ & Semimajor axis (au) & $0.148 \pm 0.004$ \\
\hline$M_{P}$ & Mass $\left(M_{\oplus}\right)$ & $27_{-12.6}^{+14}$ \\
\hline$R_{P}$ & Radius $\left(R_{\oplus}\right)$ & $6.12 \pm 0.10$ \\
\hline$\rho_{P}$ & Density (cgs) & $0.65_{-0.30}^{+0.34}$ \\
\hline$g_{P}$ & gravity(cgs) & $689.96_{-322.20}^{+360.87}$ \\
\hline$T_{P}$ & Time of periastron $\left(\mathrm{BJD}_{\mathrm{TDB}}\right)$ & $2458113.93994_{-0.00039}^{+0.00039}$ \\
\hline$T_{\text {eq }}$ & Equilibrium Temperature (K) & $886.35_{-17.10}^{+17.36}$ \\
\hline$T_{23}$ & Total Eclipse duration (hr) & $2.92_{-0.04}^{+0.04}$ \\
\hline$T_{14}$ & Total duration (hr) & $3.91 \pm 0.02$ \\
\hline \multicolumn{3}{|c|}{ Other Parameters: } \\
\hline$u_{1}$ & linear limb-darkening coeff & $0.36^{\mathrm{a}}$ \\
\hline$u_{2}$ & quadratic limb-darkening coeff & $0.29^{\mathrm{a}}$ \\
\hline$q_{1}$ & $\left(u_{1}+u_{2}\right)^{2}$ & $0.42^{\mathrm{a}}$ \\
\hline$q_{2}$ & $u_{1}\left(2\left(u_{1}+u_{2}\right)\right)^{-1}$ & $0.28^{\mathrm{a}}$ \\
\hline$\gamma_{\text {PARAS }}$ & Systemic velocity $\left(\mathrm{Km} \mathrm{s}^{-1}\right)$ & $1.2588 \pm 0.0025$ \\
\hline
\end{tabular}

Note.

${ }^{\text {a }}$ Limb-darkening coefficients obtained by interpolating the table of Claret \& Bloemen (2011).

making them an interesting class of exoplanets meriting further scrutiny.

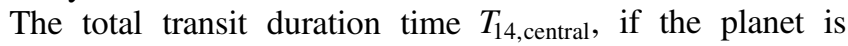
passing in front of the stellar disk center, should be $\sim 6 \mathrm{hr}$ using the equations of Seager \& Mallén-Ornelas (2003). However, fitting the light curve (Section 7) gives $T_{14}=3.91 \mathrm{hr}$, and an impact parameter of $b=0.85$, suggesting that the planet is passing closer to the poles of the stellar disk. This indication of a grazing transit scenario likely results in the $\sim 2 \%$ probability of an EB in the VESPA output. However, the RV observations from PARAS conclusively rule out the EB scenario and confirm the planetary nature of the source.

We also estimate the internal composition of EPIC 211945201b using irradiated planetary composition models of Fortney et al. (2007) and Lopez \& Fortney (2014). These are two layer partitioning models based on the assumption that planets are made up of a very dense core and a less dense envelope and that all of 


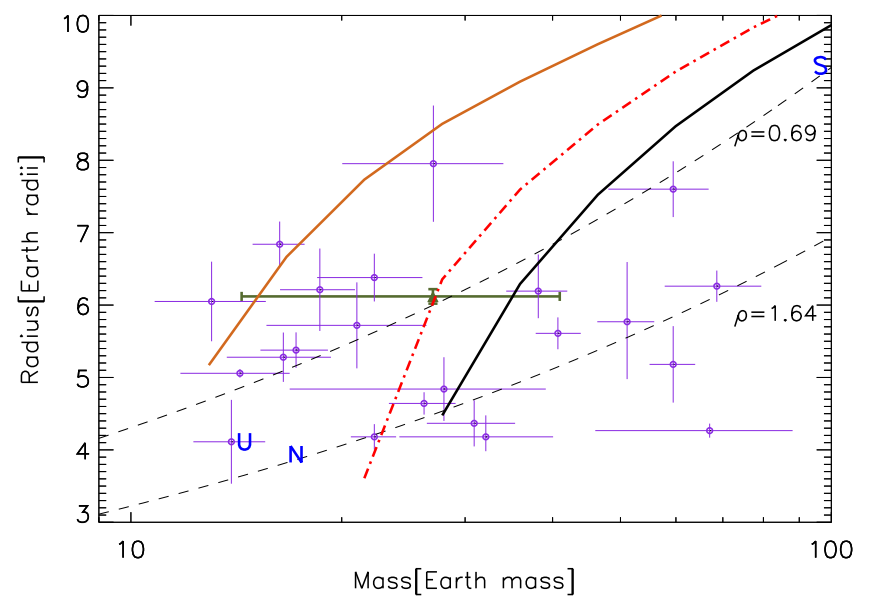

Figure 8. Mass vs. radius diagram showing our measurements for EPIC 211945201b (olive triangle). Overlaid are the theoretical curves for $3.16 \mathrm{Gyr}$ and core masses of 10 and $25 M_{\oplus}$ at 0.1 au from Fortney et al. (2007) in dark yellow and black, respectively. The dashed-dotted red line is linearly interpolated fortney model for core of $18 M_{\oplus}$ (see Section 8$)$. The dashed black lines from top to bottom are isodensity curves for Saturn \& Neptune density. The magenta colored open circles represents the 22 super-Neptunes $\left(4.0 \leqslant R_{\oplus} \geqslant 8.0,10.0 \leqslant M_{\oplus} \geqslant 70.0\right)$ whose mass and radius are determined with at least $50 \%$ precision (from http://exoplanet.eu/, Schneider et al. 2011 \& NASA exoplanet Archive, Akeson et al. 2013). The blue colored U, N, and S represents here Uranus, Neptune, and Saturn, respectively.

the heavy elements are concentrated in the core while the lighter elements are part of envelope. In Figure 8, we plot our results, along with the synthetic models used to estimate the core and envelope masses. The models chosen here are based on our final parameters for the EPIC 211945201 system-at an age of $3.16 \mathrm{Gyr}$ and 0.1 au separation, with core masses of 0 , 10, and $25 M_{\oplus}$-where we interpolate between 10 and $25 M_{\oplus}$ core mass models. We determine the heavy element content to be about $60 \%-70 \%$ of the total mass. The non-irradiated models of Mordasini et al. (2012) and Jin et al. (2014) were also used to find the heavy element fraction and the results found to be consistent with $60 \%-70 \%$. However, we caution that these values have high uncertainties given our errors on the planet density, and should be considered preliminary estimates until better mass measurements are obtained.

\section{Summary}

We find strong evidence of a transiting sub-Saturn (or superNeptune) around EPIC 211945201. Previous work had deemed this a PC, which we validate with VESPA analysis. We found the FPP for the planetary hypothesis to be $>1 \%$, with a $\sim 2 \%$ probability that the system is an EB. In order to resolve the situation, we conducted a campaign of high-resolution RV observations using the PARAS spectrograph. These RV measurements showed low dispersion over a long time baseline, eliminating the EB scenario and confirming the planetary nature of the candidate. By simultaneously modeling the RV and $K 2$-photometry data, we derive a radius and mass of $6.12 \pm 0.1 R_{\oplus}$ and $27_{-12.6}^{+14} M_{\oplus}$ for the companion. We then used the models of Fortney et al. (2007) and Lopez \& Fortney (2014) to make a notional prediction of the core mass, finding a substantial projected heavy element fraction of $60 \%-70 \%$, although more precise mass measurements are necessary to confirm this heavy element content. EPIC 211945201b remains interesting as it straddles the transition regime between ice giants and gas giants. The detection of similar exoplanets will continue to hone our understanding of the formation and distribution of these worlds that have no analog in our solar system and yet seem to abound in the nearby universe.

Future Works: The accurate mass of EPIC 211945201b can be determined with more precise RV measurements using HARPS (Pepe et al. 2003) or the newly commissioned HPF (Mahadevan et al. 2014) spectrographs. This will provide clearer insight into the internal structure of the planet. The source is also a good target to study the star planet alignment ( $\mathrm{R}-\mathrm{M}$ effect) as the host star is relatively bright.

The PARAS spectrograph is fully funded and being supported by Physical Research Laboratory (PRL), which is part of Department of Space, Government of India. We would like to thank Director, PRL for his support. We acknowledge the help from Vishal Shah, Kapil Kumar, Kevikumar Lad, Ashirbad Nayak and Mount Abu Observatory staff for their support during observations. We acknowledge the use of SIMBAD database operated at the CDS, Strasbourg, France. We also acknowledge the support from ExoFOP users, who willingly share the follow-up observational data with the community. This research has made use of the NASA Exoplanet Archive, which is operated by the California Institute of Technology, under contract with the National Aeronautics and Space Administration under the Exoplanet Exploration Program; and the Extrasolar Planets Encyclopaedia (exoplanet.eu). We are also thankful to Francesco Pepe (Geneva Observatory) for providing G2 stellar mask which is used for RV reduction.

Software: VESPA (Morton 2012, Morton 2015), PARAS PIPELINE (Chakraborty et al. 2014), PARAS SPEC (Chaturvedi et al. 2016a, 2016b), ISOCHRONES (Morton 2015), PYANETI (Barragán et al. 2016, 2017).

\section{ORCID iDs}

Abhijit Chakraborty (1D https://orcid.org/0000-0002-3815-8407 Arpita Roy (iD https://orcid.org/0000-0001-8127-5775 Rishikesh Sharma (i) https://orcid.org/0000-0001-8983-5300 Suvrath Mahadevan (iD https://orcid.org/0000-00019596-7983

\section{References}

Aigrain, S., Parviainen, H., \& Pope, B. J. S. 2016, MNRAS, 459, 2408 Akeson, R. L., Chen, X., Ciardi, D., et al. 2013, PASP, 125, 989

Baglin, A., Auvergne, M., Boisnard, L., et al. 2006, in 36th COSPAR Scientific Assembly, 3749

Baranne, A., Queloz, D., Mayor, M., et al. 1996, A\&AS, 119, 373

Barragán, O., Gandolfi, D., \& Antoniciello, G. 2017, pyaneti: Multi-planet Radial Velocity and Transit Fitting, Astrophysics Source Code Library, ascl:1707.003

Barragán, O., Grziwa, S., Gandolfi, D., et al. 2016, AJ, 152, 193

Barros, S. C. C., Demangeon, O., \& Deleuil, M. 2016, A\&A, 594, A100

Bayliss, D., Hartman, J. D., Bakos, G. Á., et al. 2015, AJ, 150, 49

Bonomo, A. S., Sozzetti, A., Lovis, C., et al. 2014, A\&A, 572, A2

Borucki, W. J., Koch, D., Basri, G., et al. 2010, Sci, 327, 977

Bouchy, F., Díaz, R. F., Hébrard, G., et al. 2013, A\&A, 549, A49

Bouchy, F., Pepe, F., \& Queloz, D. 2001, A\&A, 374, 733

Chakraborty, A., Mahadevan, S., Roy, A., et al. 2014, PASP, 126, 133

Chaturvedi, P., Chakraborty, A., \& Anandarao, B. G. 2016a, arXiv:1610.07235

Chaturvedi, P., Chakraborty, A., Anandarao, B. G., Roy, A., \& Mahadevan, S. 2016b, MNRAS, 462, 554

Claret, A., \& Bloemen, S. 2011, A\&A, 529, A75 
Crossfield, I. J. M., Ciardi, D. R., Petigura, E. A., et al. 2016, ApJS, 226, 7 Cutri, R. M., Skrutskie, M. F., van Dyk, S., et al. 2003, 2MASS All Sky Catalog of Point Sources

Cutri, R. M., Skrutskie, M. F., van Dyk, S., et al. 2013, yCat, 2246, 0

Díaz, R. F., Almenara, J. M., Santerne, A., et al. 2014, MNRAS, 441, 983

Dotter, A., Chaboyer, B., Jevremović, D., et al. 2008, ApJS, 178, 89

Dressing, C. D., Vanderburg, A., Schlieder, J. E., et al. 2017, AJ, 154, 207

Fortney, J. J., Marley, M. S., \& Barnes, J. W. 2007, ApJ, 659, 1661

Gaia Collaboration, Katz, D., Antoja, T., et al. 2018, arXiv:1804.09380

Hodapp, K. W., Jensen, J. B., Irwin, E. M., et al. 2003, PASP, 115, 1388

Howell, S. B., Sobeck, C., Haas, M., et al. 2014, PASP, 126, 398

Huber, D., Bryson, S. T., Haas, M. R., et al. 2016, ApJS, 224, 2

Jin, S., Mordasini, C., Parmentier, V., et al. 2014, ApJ, 795, 65

Kipping, D. M. 2013, MNRAS, 435, 2152

Kovács, G., Zucker, S., \& Mazeh, T. 2002, A\&A, 391, 369

Lopez, E. D., \& Fortney, J. J. 2014, ApJ, 792, 1

Luger, R., Agol, E., Kruse, E., et al. 2016, AJ, 152, 100

Mahadevan, S., Ramsey, L. W., Terrien, R., et al. 2014, Proc. SPIE, 9147, 91471G

Mandel, K., \& Agol, E. 2002, ApJL, 580, L171

Mayo, A. W., Vanderburg, A., Latham, D. W., et al. 2018, arXiv:1802.05277

Mordasini, C., Alibert, Y., Georgy, C., et al. 2012, A\&A, 547, A112
Morton, T. D. 2012, ApJ, 761, 6

Morton, T. D. 2015, VESPA: False Positive Probabilities Calculator, Astrophysics Source Code Library, ascl:1503.011

Pepe, F., Mayor, M., Galland, F., et al. 2002, A\&A, 388, 632

Pepe, F., Rupprecht, G., Avila, G., et al. 2003, Proc. SPIE, 4841, 1045

Perruchot, S., Kohler, D., Bouchy, F., et al. 2008, Proc. SPIE, 7014, 70140J

Petigura, E. A., Crossfield, I. J. M., Isaacson, H., et al. 2018, AJ, 155, 21

Petigura, E. A., Schlieder, J. E., Crossfield, J. J. M., et al. 2015, ApJ, 811, 102

Petigura, E. A., Sinukoff, E., Lopez, E. D., et al. 2017, AJ, 153, 142

Piskunov, N. E., \& Valenti, J. A. 2002, A\&A, 385, 1095

Pope, B. J. S., Parviainen, H., \& Aigrain, S. 2016, MNRAS, 461, 3399

Queloz, D., Henry, G. W., Sivan, J. P., et al. 2001, A\&A, 379, 279

Roy, A., Chakraborty, A., Mahadevan, S., et al. 2016, Proc. SPIE, 9908, 99086R

Schneider, J., Dedieu, C., Le Sidaner, P., Savalle, R., \& Zolotukhin, I. 2011, A\&A, 532, A79

Seager, S., \& Mallén-Ornelas, G. 2003, ApJ, 585, 1038

Torres, G., Fressin, F., Lissauer, J. J., et al. 2011, ApJ, 727, 24

Vanderburg, A., \& Johnson, J. A. 2014, PASP, 126, 948

Wright, J. T., Roy, A., Mahadevan, S., et al. 2013, ApJ, 770, 119

Yu, L., Crossfield, I. J. M., Schlieder, J. E., et al. 2018, arXiv:1803.04091 